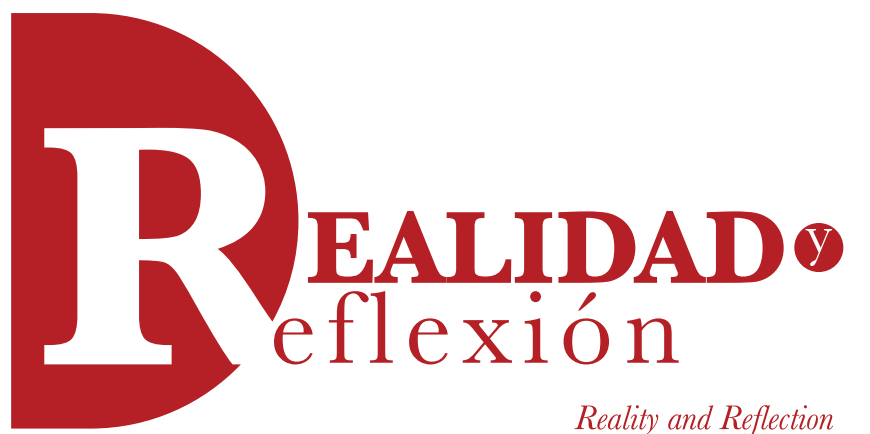

ISSN 1992-6510

Año 16, N 43, San Salvador, El Salvador, Centroamérica. Revista Semestral Enero-Junio 2016

YEAR 16, N 43, SAN SALVADOR, El SALVADOR, CENTRAL AMERICA. SEMESTRAL JOURNAL JANUARY-JUNE 2016

\title{
El Arte como recurso alternativo para la reinserción y rehabilitación de los internos en centro carcelarios
}

$$
\begin{gathered}
\text { Art as an alternative resource for reintegration and } \\
\text { Rebabilitation Opportunities of Inmate Population } \\
\text { in the prison system }
\end{gathered}
$$

\section{RESUMEN}

Roberto Vladimir Carbajal

Máster en Investigación
Docente investigador

Facultad de Ciencias Sociales UFG

rcarbajal@ufg.edu.sv

El presente artículo plantea la incorporación del arte como parte de un plan integral en la rehabilitación y reinserción del recluso, pues consideramos el hecho artístico como una oportunidad de desarrollar la sensibilidad y otros procesos de cognición para ayudar a la población interna a fin de devolverles la confianza, la integridad y liberarla de estados de tensión y frustración.

Se plantea un plan de acción que se podría desarrollar desde la fase de confianza hasta la de adaptación, además de entender este proceso como parte de un todo que conduzca a una política nacional de rehabilitación y reinserción, de la mano con otros sectores que pudieran involucrarse, tales como la empresa privada, las instituciones de educación superior, las escuelas de arte y otras que sumen esfuerzos para lograr en un futuro una sociedad más indulgente y comprensiva.

Palabras clave: arte, educación artística, rehabilitación, reinserción social.

\section{ABSTRACT}

This article discusses the incorporation of Art as part of a comprehensive plan for the rehabilitation and reintegration of the prisoner as if we see the artistic event as an opportunity to develop sensitivity and other cognitive processes in order to contribute to the inmate population to restore confidence, integrity and psychically set free from a state of tension and frustration.

One action plan could be developed from the stage of confidence to adapt well to understand this process as part of a whole arises involving a national policy on rehabilitation and reintegration of hand with other sectors that may be involved such as the company private institutions of higher education, art schools and others that join efforts to have a more lenient future society.

Keywords: art, art education, rehabilitation and social reintegration. 


\section{Introducción}

La preocupación por el incremento de la violencia en los países de Centroamérica y en particular en El Salvador, azotados por el problema del narcotráfico y la presencia de las maras, es creciente "y si bien la respuesta contra este flagelo podría ser no sólo la cultura, la cultura es una parte importante de la respuesta", sostuvo Melguizo, un comunicador nacido y criado en la Comuna XIII, uno de los barrios más conflictivos de Medellín y colaborador de la Red Latinoamericana de Arte para la Transformación Social. Para Melguizo, la clave está en detectar las organizaciones culturales que nacen espontáneamente en las comunidades y apoyarlas desde el Estado para que se conviertan en un elemento de integración de las juventudes vulnerables y en facilitadoras de la convivencia.

A diario, en El Salvador vivimos la realidad de darnos cuenta de más de 10 muertes por día, lamentable situación que mantiene la atención y preocupación de todos los sectores. Algunos de estos abogan por una solución que implique incrementar el número de policías en la calles; otros, de llevar a cabo programas de prevención. Incluso se inició una discusión sobre la posibilidad de implementar un estado de excepción, tras un el asesinato de 11 empleados de una empresa eléctrica, un hecho que conmocionó al país y que según investigaciones preliminares fue ordenado desde un recinto penitenciario.

Lo cierto es que si echamos la mirada a la población reclusa que ronda las 30,000 personas, según datos de la Dirección General de Centros Penales, ésta casi cuadruplica la capacidad de las cárceles, lo cual también es un detonante que genera violencia por el hacinamiento, la falta de programas de reinserción, de recreación y esparcimiento en dichos centros.

Este artículo plantea la situación preocupante del incremento de la violencia y el crimen y la casi nula oportunidad de reinserción o rehabilitación por parte de los reclusos ante una sociedad cada vez más limitada en oportunidades para este sector de la población.

\section{Oportunidades de reinserción}

De acuerdo a datos de la Dirección General de Centros Penales, DGCP, la población reclusa en E1 Salvador ronda los 30,000 privados de libertad, según registros hasta julio 2015, lo que casi cuadruplica la capacidad que es de 8,490 internos.

De esta población, 20,153 reclusos ya han sido condenados por los tribunales de justicia, por lo que ya cumplen sus penas dentro del sistema carcelario.

Para los que aún están siendo procesados en el sistema judicial, sus detenciones son provisionales, pues esperan una sentencia.

Señala el informe que del total de condenados, 9,292 son homicidas; otros 4,476 son extorsionistas; 4,302 fueron sentenciados por robo y 2,021 fueron condenados por delitos relacionados con el tráfico de drogas.

Además, 8,495 internos cumplen penas que oscilan entre los 6 y los 10 años de prisión; 7,759 permanecerán en la cárcel entre 11 y 20 años; 2,915 convictos purgan sentencias que van de 31 a 50 años y 1,274 reos tienen penas mayores de 51 años. 
La mayoría de los prisioneros en los centros penales tiene bajos niveles educativos, revela el informe.

De la totalidad de la población reclusa, el 38 por ciento apenas logró terminar la educación primaria y un 34 por ciento cursó hasta el noveno grado de secundaria.

Los reos que son bachilleres representan el 18 por ciento de toda la población reclusa, y solo 446 convictos (dos por ciento) cuentan con algunos estudios de educación superior o poseen un título universitario.

Centros Penales señala en su informe que la mayoría de los delincuentes encarcelados son jóvenes en edades productivas.

Un buen número de internos $(11,276)$ tienen entre 26 y 35 años de edad; seguido del otro rango de 18 y 25 años de edad $(7,671)$. Un total de 4,846 internos tiene edades entre 36 y 45 años.

El documento además detalla que 19,277 cumplen sus penas dentro del sistema carcelario y 876 gozan de las fases de confianza o semilibertad.

De los que todavía están siendo procesados judicialmente y que se encuentran dentro de un penal, a 81 reos se les cumplió el período de la detención provisional el pasado mes de agosto de 2015; a otros 131, en septiembre; a 84 más, en octubre próximo, y a 121 más, en noviembre. También el informe revela que sólo el 15 por ciento de la población reclusa; es decir, 4,070 internos son reincidentes; lo anterior significa que ya habían sido capturados por delinquir en distintas oportunidades. Para el resto, 22,442 reos, es la primera vez que se encuentran recluidos en un centro penal.

Para esta población reclusa son escasas las oportunidades de reinserción y de uso del tiempo mientras están recluidos, pese a que se cuenta con talleres de oficios varios, tales como panadería, sastrería, carpintería y otros, además de programas de educación formal donde muy pocos reclusos logran culminar su escuela secundaria.

En este contexto, el arte podría contribuir exitosamente a la reinserción. En la cárcel de Bollate, de Milán,Italia,Thomas Louvat comprobó que las personas detenidas, a causa del casi total aislamiento y separación de la sociedad libre, viven situaciones que pueden generar bloqueos físicos y mentales. Louvat plantea que a veces estos bloqueos pueden degenerar en una dificultad de expresarse y hasta el miedo y rechazo hacia la sociedad exterior. La privación de libertad tiene consecuencias negativas sobre las personas, sobre la sociedad en su conjunto y el funcionamiento del sistema penitenciario; y dificulta tomar iniciativas y responsabilizar a las personas. Estas consecuencias del encierro bloquean los procesos dinámicos de rehabilitación.

Con estos antecedentes se creó el proyecto ESTIA, con el apoyo del programa Sócrates de la Unión Europea, y que pronto encontró eco en la cárcel de Les Baumettes, en Marsella, Francia y en la cárcel de Quatre Camins, en Barcelona, España. Dentro de sus objetivos plantea desarrollar la capacidad de expresión y comunicación, estimular a los participantes en la observación y el análisis de la realidad interior y exterior. A partir de ahí, expresar y comunicar ideas, historias y problemáticas; a su vez, favorece 
la comunicación interior/exterior contribuyendo a desmontar los estereotipos y los prejuicios existentes en la sociedad libre, acerca de las personas detenidas y de la vida carcelaria.

Algo parecido ocurrió en el penal de San Miguel (2009) cuando uno de sus reclusos, egresado del Centro Nacional de Artes, se propuso formar un grupo teatral con reos, en su mayoría expandilleros, con quienes trabajó y realizaron actuaciones en el mismo reclusorio y en otros recintos como la Universidad de El Salvador y Centro Regional de Oriente. Este grupo se llama Liberación y crea sus propias piezas de teatro, basadas en la prevención de la violencia, de pandillas y acerca de la realidad que viven a diario en la cárcel.

Otra iniciativa es el taller de creación literaria desarrollado por la poetisa Silvia Elena Regalado, directora de la Casa del Escritor. Dicho taller se lleva a cabo en el Centro de Readaptación para mujeres de Ilopango y promueve la creatividad e imaginación por medio de la expresión literaria que permite a las internas liberar sus pensamientos, sus sentimientos y las experiencias de sus condiciones como internas y de la realidad que viven diariamente.

Con los antes expuesto, se plantea una acciónplan en donde se ve al teatro como medio de expresión y comunicación a través del arte, la psicoterapia y la enseñanza-aprendizaje.

La función de la expresión como acción educativa, le ayuda al sujeto a adquirir confianza en sí mismo y hacerlo cada vez más consciente de su propia capacidad de comunicación. Pero, además, la práctica de la expresión actúa también como soporte de la alfabetización estética. La práctica de la expresión ha de estar integrada en todas las áreas curriculares, ya que es necesario trabajar todas las dimensiones de la persona (emocional, relacional, corporal), y sólo las cognitivas. La expresión ha de constituir la base de los métodos activos y el espacio donde el saber ser predomina sobre el saber y el saber hacer.

La expresión permite entrar en relación con sujetos de comportamientos irregulares y difíciles. Así se pueden resolver problemas derivados de la incomunicación (autismo, soledad, bloqueos, etc.); superar conflictos personales (desinhibición, estimulación); mejorar las capacidades, lograr la autoconfianza la afectividad, la adquisición de seguridad, etc. Tenemos tendencia a conservar la memoria de las experiencias traumáticas en nuestro cuerpo. La expresión permite entonces liberar determinadas tensiones o frustraciones $y$ sustituirlas por vivencias positivas y estimulantes.

Los productos de la expresión pueden resultar formas artísticas o manifestaciones que son testimonio de un ideal del estilo propio de un individuo, de una cultura o de una época. En este caso estaríamos en la acción artística. También se entiende por expresión artística el modo de expresión personal que se apoya en la aplicación precisa de habilidades y de conocimientos (saber hacer), con miras a la traducción de ideas, sentimientos y sensaciones mediante signos, sonidos, imágenes, formas, tonos, olores, palabras, sabores, o cualquier otra estructura de conjunto que suscita impresiones agradables y armoniosas, excitantes o provocadoras. La intersección de cada uno de los tres campos anteriores produce nuevas áreas de relación. Es así como el arte, aunque no se le vea como 
una solución propiamente dicha puede ser una alternativa para la reinserción de una población excluida y que merece una oportunidad en una sociedad con altos niveles de violencia.

Otro aspecto que podríamos mencionar es la falta de programas de reinserción social que existe para los reos; uno de los más destacados es el llamado programa estrella "Yo cambio", el cual surgió en 2010 por parte del Gobierno ante el incremento de los homicidios que registraba ese año, de 12 por día. El "Yo cambio" tiene cinco componentes: 1) el de las granjas penitenciarias; 2) el trabajo en apoyo a la comunidad; 3) las cooperativas de solidaridad; 4) trabajo penitenciario y 5) el proyecto "Pintando la Libertad" que será implementado en conjunto con Brasil.

Todo esto, en suma lo que busca es hacer realidad el componente de la rehabilitación. Solo para citar un ejemplo, en las granjas penitenciarias de Izalco y de Santa Ana, según la Dirección de Centro Penales, DGCP, no se observa ninguna reincidencia. Es decir, que ninguno de los reos favorecidos ha reincidido con el programa "Yo cambio". De un universo de poco o menos 780 individuos, solamente han reincidido dos. Ello arroja una tasa extremadamente baja, de las más bajas en el continente americano y en el mundo, según lo registra la DGCP. ${ }^{1}$

El programa "Yo cambio" se está desarrollando en el Centro Penal de Apanteos y busca erradicar el ocio a través de la diversificación de actividades internas del reo y aprovechar las potencialidades de cada uno. Por ejemplo, en Apanteos hay reos

1 Declaraciones del ex director de la DGCP Nelson Rauda a sociedadcivilblogspot 26-06'12 que hablan inglés a quienes se les ha fortalecido en la parte pedagógica para que puedan enseñar ese idioma. También se dan clases de francés.

Asimismo, se destaca un proyecto de alfabetización realizado por los propios privados de libertad, que también se desarrolla en Mariona, en San Vicente y en otros centros penales del país.

Como podemos ver, son programas aislados que no responden a un plan de reinserción planificado, sino que han ido surgiendo de acuerdo a la necesidad de cada centro penal. En general, existen programas que cubren las propias necesidades de los internos tales como educación, formación laboral y religiosa. Se trata de actividades que mantengan al reo entretenido y enriquezcan su espíritu, además de estimular su creatividad a través de talleres, así como también practicar el deporte. Además, existen programas especializados los cuales van dirigidos a reos que enfrentan ciertos problemas por los cuales han sido recluidos tales como drogodependencia, agresión sexual y otros.

Un factor muy importante para ubicar al interno dentro de un determinado programa es la clasificación, atendiendo a su personalidad, el historial individual, familiar, social y delictivo, ya que estos rasgos determinarán el tipo de tratamiento adecuado para él, porque le proporciona una herramienta imprescindible para su integración a la sociedad, una vez que abandona la cárcel.

Dentro de los programas con los que cuenta el Penal de Mariona para la reinserción de los reos están los siguientes: pensamiento creativo, 
habilidades sociales, control emocional, desarrollo de valores, razonamiento crítico, violencia intrafamiliar, resolución de problemas, control de la agresión sexual, control del comportamiento agresivo y control de la ansiedad.

\section{Rehabilitación y reinserción}

La rehabilitación ${ }^{2}$ está vinculada estrechamente con la noción de reinserción social, entendida como un conjunto de acciones tendientes a promover la resocialización, la incorporación social, o aceptación del conjunto de normas $\mathrm{y}$ valores del grupo social al que pertenecen los afectados. En sentido amplio, rehabilitar implica lograr el aprendizaje de un orden social y simbólico distinto, que sea funcional para la persona.

Rehabilitación como especialidad médica es el diagnóstico, evaluación, prevención y tratamiento de la incapacidad, encaminados a facilitar, mantener o devolver el mayor grado de capacidad funcional e independencia posibles.

Tal como definió la Organización Mundial de la Salud (OMS), ${ }^{3}$ rehabilitación es el conjunto de medidas sociales, educativas y profesionales destinadas a restituir al paciente la mayor capacidad e independencia posibles. El Comité de Expertos de la OMS, en la Serie de Informes Técnicos 688, de 1981, sobre incapacidad, prevención y rehabilitación, coincidió con las siguientes definiciones:

2 Aguilar Luisa, Espina Carolina." Volver a confiar caminos para la integración postcarcelaria". Instituto de Asuntos Públicos Centro de Estudios en Seguridad Ciudadana. Universidad de Chile (2011).

$32^{\circ}$ Informe del Comité de Expertos OMS, Ginebra 1968. a) La rehabilitación incluye todas las medidas destinadas a reducir el impacto de las condiciones de incapacidad y minusvalía y hacer posible que las personas incapacitadas y minusválidas alcancen la integración social.

b) El objetivo de la rehabilitación no es sólo el entrenar a personas incapacitadas y minusválidas a adaptarse a su entorno, sino también intervenir en su entorno inmediato y en la sociedad para facilitar su integración social.

c) Las personas incapacitadas y minusválidas, sus familias y las comunidades en las que viven, deben participar en la planificación y puesta en marcha de servicios relacionados con la Rehabilitación.

La rehabilitación es una especialidad que tiene como objetivo coordinar y asegurar la puesta en marcha y aplicación de todas las medidas encaminadas a prevenir o a reducir al mínimo las consecuencias funcionales, físicas, psíquicas, sociales y económicas de las deficiencias o de las incapacidades.

Ello comporta la puesta en marcha metódica de las acciones necesarias para la realización de estos objetivos, desde el comienzo de la afección hasta la reinserción del paciente en su medio ambiente y en la sociedad.

Las actividades rehabilitadoras deben desarrollarse según una relación y un orden determinados, así como en un espíritu de cooperación mutua.

Las personas y los medios deben, por tanto, estar coordinados para alcanzar un fin común. 
Reintegración o nueva adaptación de una persona en la sociedad, después de haber estado durante un tiempo al margen de ella.

La reinserción social tendría que ser la base de nuestro nuevo sistema penal, que debe buscar no castigar al delincuente, sino ayudarle a dejar los hábitos que lo llevaron a delinquir y brindarle ayuda para que se transforme en un ser humano útil a la sociedad. Para poder promover la readaptación del delincuente, se le otorga una formación integral que le permita alcanzar una vida honrada y digna a partir del momento de su libertad.

E1 concepto reinserción significa volver a encauzar al delincuente dentro de la sociedad que lo vio cometer un delito. Al ser, en efecto, el comportamiento criminal, una forma de reacción a los esquemas y valores de la sociedad, a la cual el delincuente pertenece, y que no logra aceptar. La reinserción va dirigida a obtener la responsabilidad del reo hacia él mismo y hacia la sociedad, ya sea de un mayor conocimiento de sus deberes y una mayor capacidad de resistencia a los estímulos que lo incitan al crimen, o ya sea al reconocimiento de su culpabilidad o de los errores cometidos en el pasado.

Básicamente, los medios para lograr la reinserción son aquellos enumerados en el aludido precepto magno y los que la criminología clínica aconseja. De la misma manera que los médicos tratan a los enfermos, así los técnicos penitenciarios, a través de un tratamiento individualizado, desean sanar al delincuente de esa enfermedad llamada delito.

Enorme es el valor que se atribuye a estos medios, en torno a los cuales gira prácticamente todo el tratamiento penitenciario moderno: al trabajo se le reconoce el mérito de combatir el ocio, de sacudir al detenido del aburrimiento físico y moral, de templar su cuerpo en la disciplina y apoyarlo espiritualmente, al hacerlo sentirse útil.

A la educación se le da el mérito de combatir la ignorancia, que a menudo es la causa de los errores, y de elevar el espíritu, a fin de que el hombre ya no esté sujeto a su instinto, sino a su libre albedrío. Las actividades culturales, recreativas y deportivas mejoran el nivel cultural y las condiciones físico-psíquicas de los detenidos, además de aliviar esa carga de agresividad que generalmente se acumulan en los sujetos sometidos a un régimen restrictivo de la libertad personal.

A las actividades religiosas se les reconoce el valor de confortar al preso, de infundirle resignación cristiana, de apoyarlo moralmente, de hacerle revaluar el significado del bien y de hacerle nacer el deseo de sentirse en paz consigo mismo y con la sociedad.

\section{El arte como una oportunidad de cambio}

El arte y en especial la educación artística a veces se han interpretado como unas disciplinas informales dentro de un campo de conocimientos organizado (Villamarín, 2005). Así lo concibe la tradición heredada del movimiento pedagógico de Pestalozzi: ${ }^{4}$

La enseñanza del arte es considerada únicamente como una destreza en el desarrollo de las habilidades motrices, pero la enseñanza artística también incluye

\footnotetext{
4 John Henrich Pestalozzi, pedagogo suizo, reformador de la pedagogía tradicional indicó la necesidad de libertad en la educación del niño
} 
conocimientos relacionados con el desarrollo sensitivo, la autoexpresión, las funciones psíquicas como memoria, percepción, atención, etc., y los procesos cognitivos como pensamiento, motivación y razonamiento.

Estos aspectos son precisamente las carencias que sufren todas las personas que viven largos periodos de aislamiento, y que manifiestan una disminución en el desarrollo de su personalidad a nivel psíquico, intelectual y afectivo.

La evolución de comportamientos como la agresividad y la competición son el panorama de muchos de los internos que están en prisión, y que provienen en su mayoría de reductos con fuerte carga de violencia por parte del ambiente, la familia o las amistades. Para ellos es necesario un proyecto de educación artística orientada hacia el conflicto, en el que se contemple la construcción de una personalidad dirigida a las actitudes positivas en torno a la propia persona y a los demás.

En general, el arte puede jugar un papel muy importante a la hora de desarrollar canales de comunicación que permitan transmitir las ideas, expresar las emociones y los sentimientos presentes en todo conflicto; a su vez expresar ciertas debilidades o carencias personales. Se puede ayudar a prevenir los conflictos por medio de la intervención artística atendiendo el siguiente proceso:

1. Comunicación.

2. Búsqueda de soluciones.

3. Creación de un clima favorable.
Para lograr que dicho proceso de prevención de conflictos sea positivo es necesario favorecer en el grupo estos aspectos:

- Una comunicación abierta.

- Un ambiente de diálogo.

- Atender los intereses y las necesidades de todos.

Según Crary $^{5}$ el conflicto tiene más posibilidades de ser resuelto de forma positiva dentro de una estructura de carácter participativo, democrático y cooperativo.

El contexto del arte puede lograr que una persona que comunique un conflicto o carencia personal de tipo emocional, de adaptación, de integración, etc., con la participación en un taller de arte puede superar los siguientes aspectos frente al conflicto:

- Familiarizarse con el problema.

- Aprender a resolver otros conflictos.

- Encontrar la comprensión por parte de otras personas.

- Asimilar el cambio personal.

Sabemos que el arte genera actitudes positivas ante la vida, ante la propia persona y ante los demás, que aporta valores a todos los niveles y que favorece la apertura a otras ideologías, pensamientos y actitudes. También puede ayudar a salir del pensamiento de la droga, del delito cometido o de cualquier otro hecho que le haya marcado de forma negativa al individuo, ya que actúa como elemento liberador, aumentando la sensación de calidad de vida y creando nuevas expectativas e ilusiones.

\footnotetext{
5 Profesor e investigador del arte, teoría y crítica artística Universidad de Columbia, EE. UU.
} 
La participación del interno en un taller de arte puede favorecerlo en estos aspectos:

- A nivel cultural, para que vaya adquiriendo por sí mismo habilidades de prevención y de reinserción social, ya que los factores que explican el surgimiento y el desarrollo del uso de las drogas son fundamentalmente de tipo social y cultural.

- A nivel de expresión personal, para que pueda manifestar sus necesidades expresivas por medio de la creatividad y de la capacidad de simbolización que supone el arte, ayudándole en la expansión de los sentimientos a través de un acto de carácter lúdico.

- A nivel afectivo, para que vaya ganando en confianza y pueda conseguir niveles cada vez más altos de independencia.

- A nivel relacional, para que vaya abriendo progresivamente su círculo de relaciones y sepa adaptarse sin problemas a cualquier grupo social.

- A nivel laboral,para que vaya superando su falta de experiencia, carencia de conocimientos y de responsabilidades. Apoyando su permanente decisión de salir adelante y encontrar trabajo por sí mismo.

4. Desarrollo de un programa hacia la rehabilitación y la reinserción

\subsection{El arte como rehabilitación y reinserción}

El arte y la cultura tienen una importancia primordial para romper la rutina $\mathrm{y}$ abrir horizontes más humanizados y humanizadores del contexto y de la realidad carcelaria.

A través del arte como recurso terapéutico se busca que los reclusos puedan expresarse, conocerse, dejar fluir sus sentimientos y su capacidad de comunicación con los demás, en un proceso de transformación y crecimiento personal, modelando su personalidad $\mathrm{y}$ fortaleciendo su identidad. Es por eso que se propone partir desde dos líneas fundamentales de trabajo: la primera hacia el interior, adecuar y preparar el ambiente, ayudar a curar el interior de los privados de libertad motivándoles a comenzar una vida nueva; y la segunda línea busca generar la participación de la sociedad civil en los procesos de promoción de políticas culturales, aportando al proceso de rehabilitación $\mathrm{y}$ reinserción.

El arte es una de las manifestaciones más elevadas de la actividad humana, como elemento esencial en el desarrollo y evolución del hombre, mediante la cual se expresa una visión personal y desinteresada que interpreta lo real o imaginario con recursos sonoros, plásticos o lingüísticos.

Se ha demostrado científicamente que el estudio de las bellas artes desde edad temprana, cultiva en el ser humano una sensibilidad que lo lleva a desarrollar una ética muy sólida en su vida adulta. La música, por ejemplo, nos permite la concentración. A través de escuchar música nuestra mente navega y logramos profundizar en nuestra interioridad, también despierta el sentido del oído y esto nos hace más perceptivos; y cuando la escuchamos en forma melódica nuestro sentido del ritmo se agudiza. 
La literatura desarrolla nuestro pensamiento, el lenguaje se vuelve amplio y florido, logramos transmitir nuestras ideas con suma claridad, ya que a través de la lectura nuestro vocabulario se hace más extenso.

El dibujo, la pintura o la escultura constituyen un proceso complejo en el que estudiantes reúnen diversos elementos de su experiencia para formar un todo con un nuevo significado. En el proceso de seleccionar, interpretar y reafirmar esos elementos, por ejemplo el niño nos da algo más que un dibujo o una escultura nos proporciona una parte de sí mismo: cómo pensamos, cómo sentimos y cómo vemos.

El teatro, entendiéndolo como proceso de aprendizaje y no como resultado, permite desarrollar la creatividad individual y grupal, estimula la integración en conjunto a través del juego dramático, desarrollando el vínculo y la confianza personales.

La danza promueve el trabajo en equipo y la consecuente toma de conciencia para la asimilación de valores como la solidaridad, el respeto por la diversidad, la tolerancia, la cooperación y la valoración de la propia identidad.

$\mathrm{El}$ arte en general proporciona estas ventajas:

- Enseña al estudiante ser más tolerante y abierto. Permite que los estudiantes se expresen en forma creativa.

- Promueve el trabajo individual y colectivo, aumenta la confianza en sí mismo y mejora el rendimiento académico en general.

Es importante aclarar que no se pretende hacer artistas a todos, pues unos serán simplemente receptores y otros serán ejecutantes; es decir, por ejemplo no todos los melómanos saben tocar algún instrumento musical, pero sí aprecian la música, los coleccionistas de arte no saben pintar, pero conocen de pintura; no es necesario escribir un libro para gozar del placer de la lectura; tampoco un declamador no necesariamente recitará sus propios versos.

\subsection{Plan de acción}

Como vemos, el arte tiene una amplia sucesión para poder utilizarse en diferentes áreas del quehacer diario. Por ello, se plantea incorporar el arte, especialmente el arte dramático, como recurso elemental para la reinserción y la rehabilitación del recluso.

En la primera línea, se puede llevar a cabo una intervención consistente en: ingresar a los recintos penales y ofrecer a los internos una experiencia artística a través del teatro, que busca convertir la negatividad en armonía, ecuanimidad y equilibrio, transformar lo negativo en positivo a nivel creativo, aportando una experiencia y una comprensión nuevas del mundo y de sí mismo. La idea es que el participante reflexione y mejore su autoestima para que desee y logre manifestar un cambio de vida a través de una metodología en la que los internos vivan un proceso de acercamiento al teatro que consta de cinco fases: conocimiento, creación colectiva, dramaturgia, montaje, producción y difusión. Se genera entonces un proceso social de rehabilitación a partir de las experiencias testimoniales de los participantes y se llega a una estructura dramática. 
Gracias a improvisaciones teatrales se consigue una catarsis que libera emociones que permiten un reconocimiento del error. La dramaturgia se crea paralelamente a los ensayos y el texto final es el producto de un trabajo de limpieza y selección, lo que da origen a la obra de teatro basada en los propios testimonios de los actores. Aquí identificamos la diversidad de habilidades y destrezas que pueden desarrollar los reclusos, de tal manera que podría encontrarse un buen diseñador de vestuario o un creativo literario que escriba las experiencias vividas, o aquel realizador que llevaría a escena todas esas experiencias. Luego, se pasa a una siguiente etapa en donde el recluso ha conformado un grupo de representación escénica que le permitirá proyectarse dentro del centro carcelario y la sociedad. Es así como ya una vez producido y realizado un espectáculo por los mismos internos, se crea entre sus compañeros un clima de respeto, admiración, y el reo se siente estimulado, su autoestima se eleva y desea vincularse con los demás.

A eso podríamos llamarle una terapia a través del arte teatral,e implica que sentimientos y emociones se pueden manifestar a través del arte. Si vamos más allá se produciría una catarsis por parte de los actores, pues como lo definía Aristóteles: "Catarsis se entiende como purificación emocional, corporal, mental y espiritual. Mediante la experiencia de la compasión y el miedo, los espectadores de la tragedia experimentarían la purificación del alma de esas pasiones". En este caso, el propio recluso en su rol de actor llegaría a ese estado de purificación.

Otro factor a tomar en cuenta es a quiénes se podría dirigir este proceso Se propone realizar un plan de acción donde haya una participación escalonada de la población reclusa; es decir, primero incorporar a los reos en fase de confianza para que estos, una vez se desarrollen en primer lugar como receptores de talleres artísticos, puedan enseñar a otros que se encuentran a mitad de su condena, de esta manera se podría integrar la comunicación entre reclusos y motivaría a los que están cumpliendo condena ver el desarrollo de los que están en fase de confianza. Se propone un método lancasteriano, ${ }^{6}$ donde los talleristas, que serán formados inicialmente, prepararán a los demás, y estos, a los otros. Es decir, los primeros participantes en este plan serán los reos en fase de confianza, estos enseñarían a los de la fase ordinaria, y estos a su vez, a los de fase de adaptación.

Se propone desarrollar el plan de acción de esta manera: Iniciar con la promoción del plan, donde se motivará al interno, en primer lugar; en la fase de confianza, con quien se desarrollará un taller introductorio con los siguientes componentes:

1. Conocimiento del cuerpo y la expresión individual y colectiva como medio de comunicación interpersonal a través de improvisaciones.

2. Creación colectiva, donde los participantes se integrarán como equipo de trabajo.

3. Dramaturgia, donde se partirá de la propia experiencia vivida por el reo, como un reflejo de su propia realidad para llevarla a escena.

4. Montaje, donde se desarrollarán los ensayos preparativos que conducen a la producción de una pieza teatral.

6 John Lancaster. Pedagogo inglés, quien propone una técnica pedagógica por la cual los alumnos más avanzados enseñan a los demás compañeros. 
5. Producción de una obra dramática con la incorporación de todos los elementos escenográficos (vestuario, escenografía, utilería, atrezos y otros).

6. Difusión. Ya la obra está producida y se debe hacer la difusión para hacer partícipe a la población reclusa como espectadora y que esto sirva de catarsis.

\section{Reflexiones y argumentos}

En términos generales, la situación carcelaria en nuestro país es caótica. Al inicio se apuntaba solucionar el hacinamiento, y la falta de programas de reinserción no contribuyen en la solución del problema; caemos en un círculo vicioso, pues el reo es aprehendido y llevado al centro carcelario, aparentemente ahí terminó todo, pues tiene que pagar lo que ha hecho por el delito cometido, pero al mismo tiempo, se considera una persona que ya en vida terminó su productividad, y al cumplir su condena queda libre, pero no tendrá otra opción más que volver a delinquir, pues afuera no se le ofrece ninguna oportunidad de rehacer su vida, no encuentra trabajo y, lo que es peor, la sociedad le da la espalda y hasta lo discrimina.

Es por eso, que los programas de rehabilitación y reinserción deben ejecutarse a fin de preparar al recluso a la realidad que le espera. Sin embargo, esto no puede darse aisladamente, pues se necesita de la participación de todos.

En mayo de 2012 el expresidente de la República, Mauricio Funes, pidió apoyo a los empresarios para la creación de empleos, capacitación, parques especiales de inserción laboral y planes coordinados con las alcaldías para organizar programas de prevención "con mayor eficacia". Para ello presentó cuatro iniciativas:

1. ampleos. Un pacto nacional para la creación de empleo en una alianza entre el Gobierno y los empresarios.

2. ${ }^{a}$ Formación. Protección Civil presentó un plan de formación técnica y vocacional durante nueve meses para adolescentes de 14 y 17 años en estado de riesgo y que viven en zonas de altos índices delictivos.

3. a Parques especiales de inserción laboral y cultura de paz en territorios de alta presencia de pandillas. Sus integrantes serán capacitados para insertarlos en el mercado laboral.

4. ${ }^{a}$ Coordinación. Con las alcaldías se coordinarán programas preventivos con el fin de reducir los altos índices de violencia y delitos

En lo señalado es evidente que la petición del presidente de la República constituye una forma de integración de los sectores productivos de nuestro país para llevar a cabo un plan nacional de reinserción y rehabilitación social, además de otras entidades que puedan dar su aporte y que ya lo están ofreciendo, como iglesias, organizaciones no gubernamentales, asociaciones de la sociedad civil y otras.

Otro programa recién iniciado en septiembre de 2013 es el de un Técnico en Computación, en un convenio de la Universidad Francisco Gavidia junto con la Dirección General de Centros Penales, dirigido a 30 mujeres del Centro de Readaptación para Mujeres de Ilopango. Esto forma parte de un plan piloto de impartir carreras universitarias dentro de las prisiones y se espera que vayan desarrollándose más oportunidades de 
formación para contribuir con la reinserción de los privados de libertad. Este plan se convierte en un hecho sin precedentes ya que la oportunidad de educarse es un derecho inherente al ser humano. Al final del proceso, en 2015, se tuvieron 28 alumnas graduadas con título universitario.

De esta manera se estaría tomando en cuenta el artículo 16 de la Ley Penitenciaria de El Salvador:

Las entidades de asistencia podrán diseñar $y$ desarrollar programas en favor de los internos, en todas las actividades permitidas dentro de los centros penitenciarios, pudiendo éstas ser de carácter educativo, económico, social, moral, religioso u otros autorizados por la Dirección General de Centros Penales o el Ministerio de Justicia.

Como vemos, existen algunos programas, pero se necesitan muchos más que propongan planes permanentes en una política penitenciaria que favorezca al reo en su rehabilitación y reinserción, y no solamente verlo desde el punto de vista de que el recluso esté entretenido y pase de manera productiva su tiempo de condena, sino más bien verlo como un plan articulado junto con los sectores productivos, en donde se le dé la oportunidad al interno de incorporase a este sector como fuerza laboral; y si vemos este programa como una oportunidad de formar al reo con una carrera universitaria, se articularía un plan completo en dos fases: formación y reinserción laboral, y de esta forma se le estaría preparando para que al terminar su condena pueda incorporarse fácilmente al sistema productivo y no vuelva a delinquir.

\section{Conclusiones y recomendaciones}

El arte puede ser un inicio para dar pie a diversas estrategias que conduzcan la reinserción social de la población reclusa; pero vemos el arte nada más como un goce estético o como algo que lo realizan seres con dotes y talentos especiales.

Si bien podría hacerse un esfuerzo y echar la mirada a este problema social, se puede ver como una posibilidad de integrar las unidades de arte o desarrollo estudiantil de las universidades, el Centro Nacional de Artes, las fundaciones que dedican su labor a cultivar la creación artística $\mathrm{u}$ otras organizaciones dedicadas a este rubro, para que proyecten su quehacer hacia este sector marginado, que con un estímulo y con la oportunidad de reintegrarse a la sociedad de forma productiva, pueda reivindicarse, y a futuro lograr una sociedad libre de violencia y delincuencia.

Si bien esta propuesta está enmarcada como parte de un todo; sería conveniente crear una correlación entre Centros Penales, universidades, instituciones culturales o educativas para desarrollar este plan acción y al mismo tiempo seguir proyectando los ya existentes o mejorarlos, a fin de lograr a mediano plazo resultados satisfactorios que puedan motivar a otros reos a estos planes de reinserción. A través del arte se puede lograr transformar la conducta indebida en un comportamiento apropiado. En general, veamos al arte no como una solución sino como una alternativa hacia la rehabilitación y reinserción social del reo, y de esta manera cerrar ese círculo vicioso de reincidencia. 


\section{Bibliografía}

\section{Libros}

Aprill, A, Holyday, E. (2011) Puede el arte cambiar el mundo. New York: New York University.

Crary, J. (1992). Techniques of the Observer on Vision and Modernity in the Nineteenth Century, MIT Press, 1USA

Lancaster, J. (2001). Las artes en la educación primaria. Madrid: Ediciones Morata S.L. Ministerio de Educación.

Ojeda Velásquez, J. (2012). Reinserción social y función de la pena. México D.F.: Instituto de Estudios Jurídicos de la Universidad Autónoma de México UNAM

Pestalozzi, J.H. (2003). El canto del cisne y los destinos de mi vida. Barcelona: Editorial Laertes.

Read, H. (1990). Educación por el arte. Buenos Aires: Editorial Paidós.

Villamarín Fernández, F. (2005). Intervención artística en el medio penitenciario. El arte como reinserción social. Madrid: Universidad Complutense de Madrid.

\section{Artículos}

Sánchez Pizarro, M. (2011). Creatividad, arte y arte terapia una herramienta eficaz en la escuela. Revista Clave XXI. Madrid, España.

Louvat, T. (2010). Teatro en las Cárceles. Revista dellarte. Milán, Italia.

\section{Recursos electrónicos}

Contrapunto (2010, agosto). Cárceles con triple población reclusa. Recuperado de: http://www. archivocp.archivoscp.net/index.php?option=com content\&view=article \&id=3641: noticias - de el-salvador -contrapunto \&catid=92:ultimas noticias\&Itemid=143

Louvat, T. (2010). Teatro en las Cárceles. Revista dellarte. Milán, Italia.

Marroquín, D. (2013, agosto). 30 mil reclusos saturan cárceles y bartotinas de PNC. El Diario de Hoy. Recuperado de: http://www.elsalvador. com/articulo/sucesos/casi-mil-reclusos-saturanpenales-bartolinas-pnc-35841 http://www. visionofhumanity.org/pdf/gpi/2013_Global_ Peace_Index_Report.pdf

www.sociedadcivilblogspot.com

www.economicsandpeace.org

\section{Materiales legales}

Ley Penitenciaria de El Salvador. Asamblea Legislativa de El Salvador no: 1027 D. Oficial: 85 Tomo: 335 Publicación DO: 13/05/1997 